\title{
INTELLIGENT LOW-POWER SMART HOME ARCHITECTURE
}

\author{
CORNEL POPESCU ${ }^{* 1}$, GEORGE CULEA ${ }^{2}$ \\ ${ }^{I}$ Computer Science Department, POLITEHNICA University of Bucharest, Splaiul \\ Independentei 313, Bucuresti, 060042, Romania \\ 2 "Vasile Alecsandri” University of Bacau, Calea Marasesti 157, Bacau, 600115, Romania
}

\begin{abstract}
The Smart Home technology is a house that implements a variety of physical and digital well-integrated technologies, providing a number of functionalities like control of lighting, temperature, multimedia devices, flammable gas leakage, automatic plant irrigation or alarms security using a set of hardware components connected to a development board that can be accessed remotely through an Android application. First, is presented the context and a state-of-the-art for such of applications. Second, is made a description for the architecture hardware and software of a smart home application like custom. Finally, results are highlighted and some edifying conclusions.
\end{abstract}

Keywords: intelligent control, smart home, low-power consumption

\section{INTRODUCTION}

Internet offers its users' needs and desires of their multiple facilities both common and personal. It can be said that it is in constant expansion since Hootsuite published statistics show that last year were 4.021 billion people who use the Internet, i.e. 53\% of the global population [1]. At the local level, according to the National Institute of Statistics, 11 million people have Internet access, of which 64.3\% came from urban areas and $35.7 \%$ in rural areas [2]. Given these data, we can say that people are increasingly captivated by technology in any form. From smartphone, Smart TV, smartwatches, it is time to develop technological housing, thus appearing Smart House concept. For over a century, smart home has been presented in science fiction magazines and all types of media. This was presented as a house of the future will be on the market at any time to ease daily life through automated technology that will think and act like humans. Thus, residents of such homes will not have to worry about usual activities, but will spend time with entertainment activities. The technology of smart contemporary home is different, but the rhetoric remains the same, highlighting its potential by producing scenarios which show that the technology will face a future where smart homes will automate as much of the housework. Thus, the Smart House project was first launched in the early 1980s the National Research Center of the National Association of Home Builders, together with a collection of industrial partners [3].

\subsection{Context}

As technological development increases in all areas, and housing must adapt to the requirements, growing and users. While some technologies are still in development, some elements of smart homes require large investment in time pays off and become effective in terms of maintenance costs. In recent years, the research focuses on automatic control of all aspects of electric home systems including lighting, temperature and water. Rapid response and warning systems of smart devices installed in these houses are helpful all the occupants of a house. In addition, ease of use can improve the quality of life, particularly for the elderly or disabled.

\footnotetext{
* Corresponding author, email: cornel.popescu@cs.pub.ro

(C) 2018 Alma Mater Publishing House
} 
In addition, we can say that energy is wasted and there is a growing demand for fuel, leading to depletion of natural resources, an insufficient supply of power and, ultimately, the damage to the environment. Part of this energy is wasted in our homes, hence the need for smart homes.

\subsection{Objectives}

The main objectives include:

- comfort by the possibility of controlling multiple devices via a graphical interface available on mobile devices;

- $\quad$ safety by the possibility of notice in case of fire or gas leak flammable with automatic security system arming when no human presence is detected;

- $\quad$ efficient by optimizing electricity consumption devices and the methods of producing electricity using solar energy;

- $\quad$ flexibility by the ability to access different features and remote home monitoring

In implementing the solution for the managing the features and functionality of monitoring the house, it uses a development board which can control various sensors, LEDs and motors and for the communication with the user interface and graphical mobile application easily use which allows quick and easy access to utilities offered. All data related to users, data from sensors and other necessary data are stored in a database on the development board and are transferred to the mobile device when required.

The results are given development solution of the following features: control the lighting system; control and monitoring of the climate system; automatic control of plant irrigation system in the dwelling; access control system, security and safety in case of fire or release flammable gases.

Advantages:

- Convenience: devices can be accessed remotely or from anywhere in the house;

- Customization: the installed system can be programmed according to customer needs, which is the one who chooses the number and type smart devices included;

- Security: smart homes are well-known which provides improved safety by various methods;

- $\quad$ Ease: it's quick and easy installation of the whole system;

- Efficiency: air-conditioning and lighting can be controlled for optimum energy efficiency

Disadvantages:

- Cost: some smart products can be quite expensive;

- Learning: it requires a learning time for people who are not familiar with the new technology;

- Reliability: most smart home based on the internet;

- Hackers: devices connected to the Internet are vulnerable to attacks.

\subsection{State-of-the art}

To control multiple devices is the use of a main controller to handle control. Amazon, Apple and Google have removed one device that provides a solution to the problem:

- Amazon Alexa is a system that has emerged with smart Echo box. This system allows the user to communicate with it via voice commands $[4,5]$;

- Home Kit Apple is a software framework found in operating systems iOS that allows intelligent control devices directly from compatible mobile devices with this operating system without the need for additional specific mobile applications for those devices [6];

- Google Assistant was created in response to Amazon and Alexa is a system currently in Google Home speakers, but also in mobile devices running the Android operating system [7].

All three systems can communicate with various smart devices made by manufacturers other than the systems themselves. These smart devices must be on the same Wi-Fi network to be discovered and must be compatible with these systems. Such devices include:

- Smart bulbs, which just introduced specific socket and via Wi-Fi, enabling features such as color change or intensity adjustment directly from the application or through voice commands;

- Smart sockets, which are inserted into a common outlet, and via Wi-Fi can turn on or off various appliances remotely, and can even have a program and then perform this function; 
- Smart thermostats that are physically connected to an air conditioning system and through the three temperature systems can read and even change the temperature set by the user's desire;

- Smart locks, which offers besides traditional functionality to lock the door with a key opportunity to lock it using voice commands or a mobile app menu.

\section{ARCHITECTURE AND IMPLEMENTATION OF INTELLIGENT HOME SOLUTION}

The general architecture of the proposed solution is made up of two main components:

- A development board (single-board computer) Raspberry Pi Model B + 3, comprising: a main server computer application that manages all requests from clients. A central database that stores all data necessary for correct operation with other data from various sensors, a sensor and other hardware connected directly or indirectly by means of relays or of analog-to-digital converters;

- A mobile application that provides graphical interface through which the client can access functionality and application resources using the mobile device to provide information on the development board user proximity.

\subsection{Hardware architecture}

Hardware architecture consists of several components such as: LED together with suitable resistors to limit the current values depending on the desired luminous intensity and also to protect against burning lamp; relays controlled separately from one optocoupler allows the use of hardware which requires a higher current and thus cannot be connected directly to the development; MQ135 gas sensor module, which provides the result as an analog signal, analog-to-digital converter connected to the input pin 1; ADS1115 analog-digital converter connected to the SDA and SCL pins of development of the plate that converts the signal from the gas sensor is a digital signal that can be understood by plaque development; soil moisture sensor; submersible pump powered from 5V; sensor Motion HC-SR501; fan powered by the 5V. All these components are connected to said developing Raspberry PI 3 Model B + characterized by a processor Broadcom architecture Cortex A-53 64 bit 1.4 GHz, 1GB LPDDR2 connectivity via Wi-Fi and Bluetooth, and running the operating system Raspberry Stretch $[8,9,10]$. The connection between the hardware and the development board is carried out by means of the header 40-pin GPIO [8]. The Raspberry PI 3 board is shown below in Figure 1.



Fig. 1. Raspberry PI 3 Model B basic hardware component and GPIO. 


\subsection{Software implementation}

For mobile application development was using Android platform via Android Studio development environment. This platform allows the development of mobile applications using the Java programming language and using the tools offered by Android software development kit (SDK) [11].

For the communication with the database and data sharing mobile app using PHP is a server-side scripting language widely used for web development [12]. To use PHP development board was installed XAMPP opensource platform developed by Apache friends, which is a local server via Apache distributions [13].

All the functionality offered by the solution presented requires a persistent storage medium for data. MariaDB is a management system relational database (RDBMS), open source, which is based on the SQL and guaranteeing atomicity, consistency and durability transactions [14].

To control and interrogate all the hardware used (sensors, LEDs, relays, etc.) used Python, an interpreted language, high-level, object-oriented, which enables rapid development through programming syntax very easy to use $[15,16]$.

Basic software components listed above are shown in Figure 2:



Fig. 2. Smart home software components.

In the picture above you can see how the Android app, running on the mobile device communicates bi-level application protocol HTTP using POST and GET methods to call the PHP scripts and performed by the Apache web server. These scripts can do the following operations:

- input, modification or deletion of data associated application database through SQL statements that are executed by the RDBMS MariaDB;

- $\quad$ executing Python scripts that extract data from sensors or execute instructions that modify the state of hardware.

Most of HTTP requests that must provide for PHP scripts are performed by the POST method to ensure the security of data sent. However, all HTTP requests return a message containing a success or error code with data, if required. The return code is used to notify the user if the command was completed successfully performed unsuccessfully or partially suggestive messages.

PHP files are used by Apache to interpret commands sent from the mobile app, aimed at executing the desired functionality. As can be seen almost all PHP files using the file connect.php to access the database because it plays a vital role in the proper functioning of the computer system. Also access to the hardware is done by Python files with specific libraries for existing hardware modules. 


\section{RESULTS AND DISCUSSION}

Compared with the three solutions offered by Amazon, Apple and Google, the proposed smart home solution provides a higher degree of customization and can be designed exactly to the needs of users with them. This solution provides computer and a mobile application that can remotely control home, and thus increases the flexibility of use of the product. Smart home application was designed to automate various processes of an average home, but can be extended to larger buildings. In addition, a modular nature allows adding new functionalities.

Compared with the three solutions offered by Amazon, Apple and Google, the proposed solution provides a higher degree of customization and can be designed exactly to the needs of users with them. The proposed solution provides computer and a mobile application that can remotely control home, and thus increases the flexibility of use of the product. The three solutions with the solution presented in this paper, promise a modular nature allows adding new functionalities, after initial installation.

\section{CONCLUSIONS}

In conclusion, the smart home is increasingly popular, and the functionality they provide is constantly increasing. The proposed IT solution implements all the functionality through a combination of hardware and software, allowing users:

- Control of lighting system remotely using the mobile app;

- Control and monitoring of the climate system that allows monitoring of temperature and humidity for the past few days, and setting the optimum temperature for the climate system;

- Automatic control of plant irrigation system in the dwelling without user intervention;

- Control system access, security and safety in case of fire or release of flammable gases by chance notification in case of fire or gas leak flammable with reinforcement automatic security system when detected no presence of users.

Communication between server on-board and mobile application that provides user interface is performed over HTTP, and transmitting data between the two entities is done using GET and POST methods of the same protocol. For a well-functioning information system it is necessary to store persistent information about users, and data from sensors or condition. This is done using MariaDB RDBMS software allows us to create a local database server on-board.

Fairness proposed solution has been tested on a small scale, using multiple mobile devices. The solution works fine for multiple devices connected simultaneously and component status change is visible on all connected devices.

\section{REFERENCES}

[1] https://hootsuite.com/pages/digital-in-2018, „The global state of digital in 2018” (11.06.2018).

[2] http://www.insse.ro/cms/sites/default/files/com_presa/com_pdf/tic_r2017.pdf (11.06.2018).

[3] https://www.ukessays.com/essays/information-technology/the-smart-house-project-information-technologyessay.php, „The Smart House Project Information Technology” (11.06.2018).

[4] http://st.hzcdn.com/static/econ/HouzzSmartHomeStudy2016.pdf, ,, Smart Home Study” (11.06.2018).

[5] https://www.the-ambient.com/guides/amazon-alexa-missing-manual-130 (12.06.2018).

[6] https://www.the-ambient.com/guides/apple-homekit-complete-guide-194 (12.06.2018).

[7] https://www.the-ambient.com/guides/google-home-assistant-essential-guide-146 (12.06.2018).

[8] https://www.raspberrypi.org/products/raspberry-pi-3-model-b-plus/ (14.06.2018).

[9] https://www.circuits.dk/everything-about-raspberry-gpio/ (14.06.2018).

[10] https://www.jameco.com/Jameco/workshop/circuitnotes/raspberry-pi-circuit-note.html (14.06.2018).

[11] https://developer.android.com/training/basics/network-ops/connecting (15.06.2018).

[12] http://php.net/manual/en/intro-whatis.php (15.06.2018).

[13] https://www.wpblogx.com/what-is-xampp/ (15.06.2018).

[14] https://searchdatamanagement.techtarget.com/definition/MariaDB (15.06.2018).

[15] https://www.python.org/doc/essays/blurb/ (15.06.2018).

[16] https://docs.python.org/3/index.html, ,Python 3 documentation” (15.06.2018). 